\title{
Measuring the Superfluid Fraction of an Ultracold Atomic Gas
}

\author{
Nigel R. Cooper and Zoran Hadzibabic \\ Cavendish Laboratory, University of Cambridge, J. J. Thomson Ave., Cambridge CB3 OHE, U.K.
}

(Dated: 26 October, 2009)

\begin{abstract}
We propose a method to measure the superfluid fraction of an atomic gas. The method involves the use of a vector potential generated by optical beams with non-zero angular momentum to simulate uniform rotation. The induced change in angular momentum of the atomic gas can be measured spectroscopically. This allows a direct determination of the superfluid fraction.
\end{abstract}

PACS numbers: 03.75.Kk, 67.85.-d, 37.10.Vz

Central to the understanding of the physics of degenerate Bose gases are the concepts of Bose-Einstein condensation and superfluidity [1, 2]. Bose-Einstein condensation refers to the macroscopic occupation of a single quantum state. Superfluidity refers to a set of fascinating hydrodynamic phenomena, notably persistent (dissipationless) flow.

Both phenomena admit clear quantitative definitions, allowing a Bose gas to be characterised by "condensate" and "superfluid" fractions [1, 3]. These two quantities in general take very different values. A gas of noninteracting bosons at low temperature forms a BoseEinstein condensate (BEC), but is not superfluid. In the low-temperature limit, liquid ${ }^{4} \mathrm{He}$ is both a $\mathrm{BEC}$ and a superfluid, but the condensate and superfluid fractions are markedly different, believed to be $\sim 10 \%$ and $100 \%$, respectively [1]. In $2 \mathrm{D}$ systems the superfluid fraction can be non-zero even if the condensate fraction vanishes.

For ultra-cold atomic Bose gases, the condensate fraction is readily measured through the mapping of occupation numbers in momentum space to real space by expansion imaging [4]. Characteristic signatures of superfluidity have been observed in atomic gases, notably dissipationless flow [5, 6], and the formation of quantized vortices in rotating gases 7]. However, there has been no quantitative measurement of the superfluid fraction. Such a measurement is crucial for the investigation of some of the most interesting properties of interacting Bose gases [8]: strong interactions can lead to condensate depletion without loss of superfluid fraction; the Kosterlitz-Thouless phase transition in a quasi-2D geometry is manifest by a universal jump of the superfluid density [9].

In this Letter, we describe how the superfluid fraction of an atomic gas can be measured using a light-induced vector potential [8]. Our method is closely analogous to the classic experimental method of Andronikashvili [10]. There, liquid helium is put in contact with a rotating object: the normal fluid picks up non-zero angular momentum, while the superfluid acquires no angular momentum. A measurement of the angular momentum of the fluid then allows a determination of the superfluid fraction. Here, we consider the use of an optically induced vector potential to simulate uniform rotation [8].
We show how spectroscopy can be used to measure the net change in angular momentum of the fluid, and hence the superfluid fraction. Recently, measurement of the superfluid fraction from the density profile of a rotating gas was also proposed [11]. Throughout the paper, we consider a gas of identical bosons, but the method can be extended to other situations, such as superfluidity of paired fermions.

The definition of the superfluid fraction was expressed in a form suitable for our purposes by Leggett [12]. It applies to a fluid contained in a ring-shaped vessel with a radius $R$ that is large compared to its transverse dimensions, so that the classical moment of inertia is $I_{\mathrm{cl}}=N M R^{2}$ for $N$ atoms of mass $M$. We start by adopting this assumption of geometry, but this will be relaxed at the end of the paper. The walls of the vessel are taken to rotate with angular velocity $\omega$, and the fluid allowed to come to thermal equilibrium. Under these conditions, the superfluid fraction is 12 ]

$$
\frac{\rho_{s}}{\rho} \equiv 1-\lim _{\omega \rightarrow 0}\left(\frac{\langle L\rangle}{I_{\mathrm{cl}} \omega}\right)
$$

where $\langle L\rangle$ is the average angular momentum of the fluid. A normal fluid will come to rest in the frame rotating with the walls, so that $\langle L\rangle=I_{\mathrm{cl}} \omega$ and $\rho_{s} / \rho=0$. A (perfect) superfluid is unaffected by the rotating walls, so $\langle L\rangle=0$ and $\rho_{s} / \rho=1$.

When the fluid is in equilibrium with the rotating walls it is described by the equilibrium density matrix defined by the Hamiltonian in the rotating frame [13]

$$
H_{\mathrm{rot}}=H-\boldsymbol{L} \cdot \boldsymbol{\omega} .
$$

Here, $H$ and $\boldsymbol{L}$ are the Hamiltonian and the total angular momentum in the laboratory frame. We shall show how a Hamiltonian of the form (2) can be achieved for an atomic gas, and how the resulting average angular momentum $\langle L\rangle$ can be measured so that (11) can be directly applied.

In the ring geometry, the kinetic energy in (2) can be written

$$
\frac{\boldsymbol{p}^{2}}{2 M}-\boldsymbol{r} \times \boldsymbol{p} \cdot \boldsymbol{\omega}=\frac{\boldsymbol{p}_{\perp}^{2}}{2 M}+\frac{\hbar^{2}}{2 M R^{2}} \ell^{2}-\hbar \omega \ell,
$$

where $\boldsymbol{p}_{\perp}$ is the momentum in directions perpendicular to the azimuthal direction, and $\ell$ is the angular momentum in units of $\hbar$ (therefore quantized to integer values). 
The rotation shifts the energy minimum in the angular momentum to

$$
\ell^{*}=\frac{M R^{2} \omega}{\hbar}=\frac{I_{\mathrm{cl}} \omega}{N \hbar} .
$$

This shift can be viewed as an azimuthal vector potential corresponding to a non-zero flux threading the ring.

A shift in the dispersion relation can be achieved by the use of two-photon Raman transitions to imprint vector potentials [8]. This was recently implemented [14] using two counter-propagating laser beams to couple states $m=-1,0,1$ of the $F=1$ hyperfine levels of ${ }^{87} \mathrm{Rb}$. The two-photon processes lead to a linear vector potential directed along the axis of the lasers 14, 15.

To generate an azimuthal vector potential, we consider two Laguerre-Gauss (L-G) beams [16] with different orbital angular momenta, co-propagating in the direction perpendicular to the toroidal trap. In this way, a twophoton transition imparts negligible linear momentum to the atoms, but a non-zero angular momentum, $\pm \Delta \ell$, where $\Delta \ell$ is the difference in the orbital angular momenta of the two beams. For a 3-level system [14, 15] this leads to an effective Hamiltonian

$$
\left(\begin{array}{ccc}
\frac{\hbar}{2 M R^{2}}(\ell+\Delta \ell)^{2}-\delta & \Omega_{R} / 2 & 0 \\
\Omega_{R} / 2 & \frac{\hbar}{2 M R^{2}} \ell^{2}-\epsilon & \Omega_{R} / 2 \\
0 & \Omega_{R} / 2 & \frac{\hbar}{2 M R^{2}}(\ell-\Delta \ell)^{2}+\delta
\end{array}\right)
$$

which is a matrix in the hyperfine states $m=-1,0,+1$. $\Omega_{R}$ is the two-photon Rabi frequency, $\delta=g_{F} \mu_{\mathrm{B}} B / \hbar$ is the detuning of the lasers from the Raman resonance set by the Zeeman effect of a (uniform) magnetic field $B$, and $\epsilon$ accounts for the quadratic Zeeman effect.

The energy eigenvalues for the angular motion are illustrated in Fig. 1. The lowest energy band has a minimum at a non-zero angular momentum $\ell^{*}$. To derive analytic expressions for the energies and wavefunctions in this band, we consider the limit of large Rabi frequency and develop a perturbation theory in $1 / \Omega_{R}$. We parameterise the resulting single-particle energy as

$$
E=E_{0}+\frac{\hbar^{2}}{M^{*} R^{2}}\left(\frac{\ell^{2}}{2}-\ell \ell^{*}\right) .
$$

The energy $E_{0}$ is a global shift that depends on all parameters of the optical field. A slight increase in the effective mass for the azimuthal motion is given by

$$
M^{*}=M\left(1+\frac{\sqrt{2} \hbar \Delta \ell^{2}}{M R^{2} \Omega_{R}}\right)+\mathcal{O}\left(1 / \Omega_{R}^{2}\right) .
$$

The most important effect for our purposes is the shift in the minimum of the dispersion curve to

$$
\ell^{*}=-\sqrt{2} \frac{\delta}{\Omega_{R}} \Delta \ell+\mathcal{O}\left(1 / \Omega_{R}^{2}\right)
$$

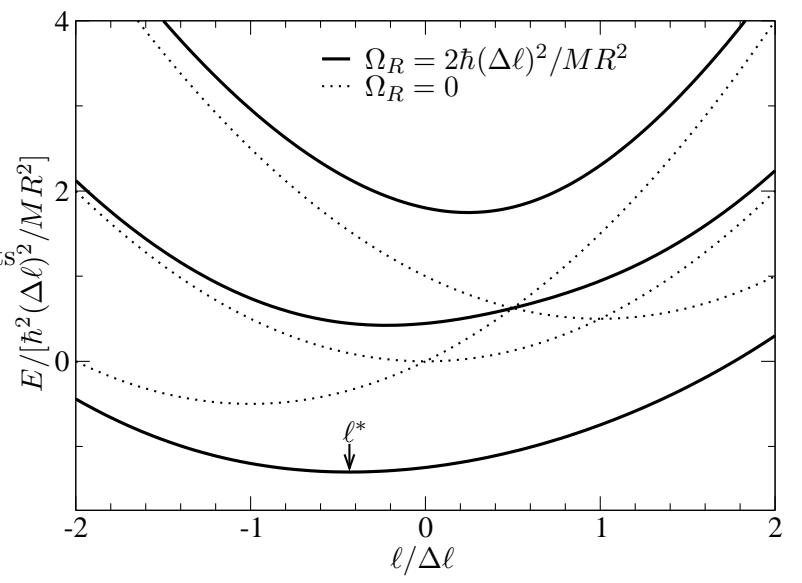

FIG. 1: Energy levels for the angular motion of an atom under the influence of two-photon Raman coupling via beams with orbital angular momentum difference $\Delta \ell$. The atoms move in a trap of radius $R$, the detuning is $\delta=0.5 \hbar(\Delta \ell)^{2} / M R^{2}, \epsilon=0$, and Rabi frequencies of $\Omega_{R}=0$ (dotted line) and $\Omega_{R}=2 \hbar(\Delta \ell)^{2} / M R^{2}$ (solid lines) are shown. The lowest band has its minimum displaced to a nonzero angular momentum, $\ell^{*}$, equivalent to the effect of an azimuthal vector potential. (Note that the smooth curves should be viewed as interpolations between the allowed integer values of $\ell$.)

This can be viewed as the introduction of an effective vector potential. In order to clarify the physical consequences of $\ell^{*}$, note that, for an atom with angular momentum $\ell$, the angular velocity when the light is on is

$$
\omega_{\text {light }} \equiv \frac{1}{\hbar} \frac{d E}{d \ell}=\frac{\hbar}{M^{*} R^{2}}\left(\ell-\ell^{*}\right) .
$$

Thus, for given $\ell$, the most significant effect of the light is to cause a constant shift in the angular velocity by $\hbar \ell^{*} / M^{*} R^{2}$. In analogy with (3, (4), it is as if the optical field causes the laboratory frame to behave as a frame of reference that is rotating with angular frequency

$$
\omega_{\mathrm{eff}} \equiv \frac{\hbar \ell^{*}}{M^{*} R^{2}} \text {. }
$$

The lowest band (5) plays the role of $H_{\text {rot }}$ (2), with an effective rotation rate (9) that can be tuned by the parameters of the optical field. Provided the splitting of the bands is large compared to the chemical potential, $\Omega_{R} \gtrsim \mu / \hbar$, all atoms are restricted to this lowest band. Then, if the position of its minimum (77) is varied sufficiently slowly that the fluid has time to come to equilibrium for the new $\ell^{*}$, a clear distinction appears between normal and superfluid components: the normal fluid will relax, and pick up a non-zero angular momentum; the superfluid will not relax, but will retain vanishing average angular momentum. This distinction provides the definition of the superfluid fraction (11).

This behaviour of the atomic gas in a toroidal trap with an azimuthal vector potential is in marked contrast 
to the case of a linear vector potential [14]. There, both superfluid and normal components must come to rest in the laboratory frame, consistent with the observations in Ref. [14]. Here, when the normal fluid comes to equilibrium at a non-zero $\ell^{*}$, it is at rest in the laboratory frame. However, the superfluid is rotating, as follows from (8). The azimuthal vector potential causes a steady superfluid flow around the ring-shaped trap. Note that for the normal fluid to come to equilibrium with the new $\ell^{*}$, it must change its angular momentum. Therefore, the trap must not be perfectly rotationally symmetric. (No fluid can come to equilibrium in a rotating container if the walls of the container are perfectly smooth.) It is an important practical feature that there is no requirement for the trap to have perfect rotational symmetry.

In the Andronikashvili experiment [10] a torsional oscillator is used to measure the moment of inertia of the fluid coupled to the oscillator, $I$. A frequency shift arises from the fluid's contribution to the energy, which in the rotating frame is $\left\langle H_{\text {rot }}\right\rangle=-\frac{1}{2} I \omega^{2}$. Here, when the light is on there is a contribution to the energy of $-\frac{1}{2} I \omega_{\text {eff }}^{2}$. One can envisage various ways in which an oscillator can modulate $\omega_{\text {eff }}$ (9) and therefore experience a frequency shift related to $I$. For example, given that $\omega_{\text {eff }} \propto \ell^{*} \propto B$, the coil that generates the Zeeman field $B$ experiences the moment of inertia as a reduction in its inductance; this could appear as a shift in the resonant frequency of an electrical circuit containing the coil. However, the total energy $\frac{1}{2} I\left(\hbar \Delta \ell / M^{*} R^{2}\right)^{2}$ is very small $(\simeq 0.1 \mu \mathrm{eV})$ making the signal small compared to typical sensitivities of current micro-mechanical or electrical oscillators.

A key element of our proposal is that, with the above light-induced vector potential, one can use spectroscopic methods to determine the average angular momentum $\langle L\rangle$. The wavefunction in the lowest band is a linear superposition of the three hyperfine levels $|\psi\rangle \equiv \sum_{m=-1,0,1} \psi_{m}|m\rangle$ with amplitudes $\left\{\psi_{m}\right\}$ which vary with $\ell$. A perturbative analysis shows that there are equal and opposite corrections to $\left|\psi_{ \pm 1}\right|^{2}$ which depend linearly on $\ell$. Thus, $\langle L\rangle$ can be obtained from a measurement of the difference in the number of particles in the states $m= \pm 1$. Using the Taylor expansion

$$
\left|\psi_{-1}\right|^{2}-\left|\psi_{1}\right|^{2} \equiv \Delta p_{0}+\Delta p^{\prime} \ell+\mathcal{O}\left(\ell^{2}\right)
$$

we can write

$$
\frac{\langle L\rangle}{\hbar N} \equiv \frac{\sum_{\ell}\left\langle n_{\ell}\right\rangle \ell}{\sum_{\ell}\left\langle n_{\ell}\right\rangle}=\frac{\Delta p-\Delta p_{0}}{\Delta p^{\prime}}
$$

where we define the fractional imbalance

$$
\Delta p \equiv \frac{N_{-1}-N_{1}}{N}=\frac{\sum_{\ell}\left\langle n_{\ell}\right\rangle\left[\left|\psi_{-1}\right|^{2}-\left|\psi_{1}\right|^{2}\right]}{\sum_{\ell}\left\langle n_{\ell}\right\rangle},
$$

with $\left\langle n_{\ell}\right\rangle$ the average number of particles with angular momentum $\ell$. Inserting (11) in (1) one finds

$$
\frac{\rho_{s}}{\rho}=1-\lim _{\ell^{*} \rightarrow 0}\left(\frac{\Delta p-\Delta p_{0}}{\ell^{*} \Delta p^{\prime}}\right)+\mathcal{O}\left(\mu / \hbar \Omega_{R}\right),
$$

where the corrections arise from the approximation (10), which is accurate provided the atoms are in the parabolic region of the lowest band.

In the limit of large $\Omega_{R}$, the wavefunction of the lowest band is $\left(\psi_{-1}, \psi_{0}, \psi_{1}\right)=1 / 2(1,-\sqrt{2}, 1)$ for all $\ell$. Computing perturbative corrections to order $1 / \Omega_{R}^{2}$ in $\left|\psi_{m}\right|^{2}$, we find $\Delta p_{0} \simeq\left(\delta / \Omega_{R}^{2}\right)\left[\sqrt{2} \Omega_{R}-\hbar(\Delta \ell)^{2} /\left(2 M R^{2}\right)-2 \epsilon\right]+$ $\mathcal{O}\left(1 / \Omega_{R}^{3}\right)$, and $\Delta p^{\prime}=\sqrt{2} \hbar \Delta \ell /\left(M R^{2} \Omega_{R}\right)+\mathcal{O}\left(1 / \Omega_{R}^{2}\right)$. Combining this with (7) we obtain

$$
\frac{\rho_{s}}{\rho}=1-\lim _{\delta \rightarrow 0}\left(\frac{\Delta p-\Delta p_{0}}{2 \hbar \delta(\Delta \ell)^{2} /\left(M R^{2} \Omega_{R}^{2}\right)}\right)+\mathcal{O}\left(1 / \Omega_{R}\right),
$$

The limit $\omega \rightarrow 0$ in (1), replaced here by $\omega_{\text {eff }} \propto \delta \rightarrow 0$, is discussed further below.

Eqns. (13, 14) show how a spectroscopic measurement of the populations $N_{m}$ can lead to a direct measurement of the superfluid fraction. That there is a connection between these quantities is a central result of this paper.

To distinguish a normal fluid from superfluid, the fractional population difference $\Delta p$ (12) must be measured with an absolute accuracy of order

$$
\frac{2 \hbar(\Delta \ell)^{2} \delta}{M R^{2} \Omega_{R}^{2}}
$$

This expression was derived for $\delta / \Omega_{R} \ll 1$. In Fig. 2 we show the expected fractional change in occupation for a normal fluid, with angular momentum centred on $\ell^{*}$, computed for arbitrary $\delta / \Omega_{R}$. This is shown for parameters which for ${ }^{23} \mathrm{Na}$ would correspond to $R=10 \mu \mathrm{m}$, $\Omega_{R} \simeq 2 \pi \times 4.4 \mathrm{kHz}$, and $\Delta \ell=10$ (two beams of orbital angular momentum 5). In this case, $\Delta p$ must be measured to an absolute accuracy of about $3 \%$. The required relative accuracy to distinguish a normal fluid $\left(\Delta p-\Delta p_{0} \neq 0\right)$ from a superfluid $\left(\Delta p=\Delta p_{0}\right)$ is $\left(\Delta p-\Delta p_{0}\right) / \Delta p_{0}$, and is about $10 \%$ in the linear regime $\delta / \Omega_{R} \lesssim 0.25$ in Fig. 2. This relatively small signal poses a moderate experimental challenge. It is important to stress that it relies only on the measurement of fractional occupations of different states. It is therefore insensitive to systematic uncertainties in the absolute atom number determination, and statistical errors can be reduced by averaging over many shots.

Above we used $\Delta \ell=10$ as a currently realistic experimental value, but the signal can be increased by increasing $\Delta \ell$. Since we require $\Omega_{R} \gtrsim \mu / \hbar$ for atoms to be in the parabolic region of the lowest band, it is instructive to write (15) in the form $\left(\mu / \hbar \Omega_{R}\right)\left(4 \delta / \Omega_{R}\right)(\Delta \ell \xi / R)^{2}$, where $\xi$ is the healing length. All three terms in this expression can in principle be close to unity. One limitation to $\Delta \ell$ arises from the fact that the expression (14) applies in the limit $\omega_{\text {eff }} \propto \delta \rightarrow 0$, so that the imposed rotation is sufficiently small that the superfluid does not change its angular momentum. This will fail if the resulting velocity of the superfluid, $R \omega_{\text {eff }}=\hbar \ell^{*} /\left(M^{*} R\right)$, becomes larger than the superfluid critical velocity, which is $\sim \hbar /(M \xi)$. 


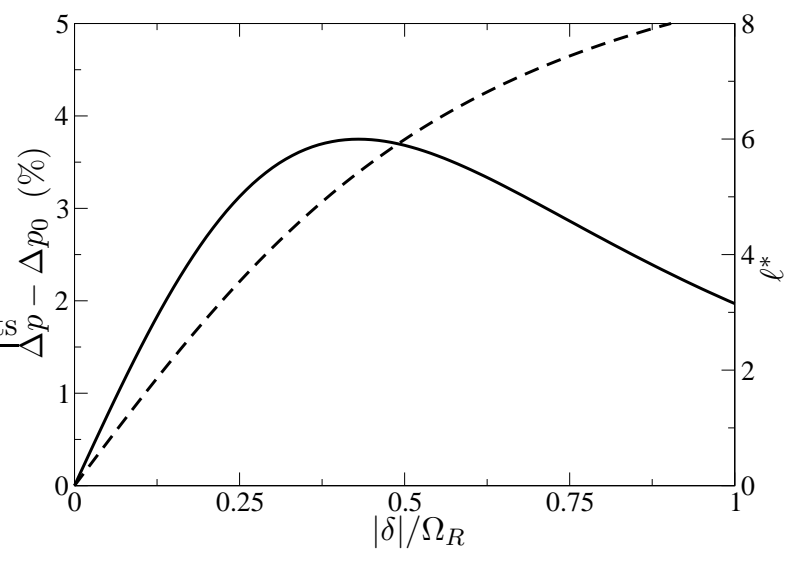

FIG. 2: Angular momentum $\ell^{*}$ at the bottom of the band (dashed line) and change in particle imbalance $\Delta p-\Delta p_{0}$ (solid line) as a function of $\delta / \Omega_{R}$ for a normal fluid (i.e. centred on $\left.\ell^{*}\right)$ for $\Omega_{R}=1000 \hbar / M R^{2}, \Delta \ell=10, \epsilon=0$. This illustrates the precision required to distinguish a normal fluid (here $\Delta p-$ $\left.\Delta p_{0} \sim 3 \%\right)$ from a perfect superfluid $\left(\Delta p-\Delta p_{0}=0\right)$.

If this condition is violated, the superfluid will relax (vortices will enter the system) and acquire non-zero angular momentum. The condition for stability of the superfluid flow requires $\Delta \ell$ to be less than $\sim\left(\Omega_{R} / \delta\right) R / \xi$ for $\delta \ll \Omega_{R}$ (7), and $\sim R / \xi$ otherwise. (Note that typically $\xi \leq 0.5 \mu \mathrm{m}$.) As (15) suggests, if $\Delta \ell$ is limited by practical reasons, the signal will generally be larger for lighter species, which for typical experimental parameters have lower density, and hence larger $\xi$.

Experimentally, a large spectroscopic signal which qualitatively distinguishes normal and superfluid components can be observed by a "projective measurement": Suppose that the system is in equilibrium in the parabolic band with $\Omega_{R} \gg \mu / \hbar \approx \delta$. If we then reduce $\Omega_{R}$ to $\sim \delta$ on a time scale short compared to the relaxation time but long compared to $1 / \Omega_{R}$, the superfluid and the normal fluids will remain centred at $\ell=0$ and $\ell=\ell^{*}$, respectively, but the difference in their spin composition will be greatly enhanced (see Fig. 1). However the quantitative extraction of the superfluid fraction would in this case require further analysis.

The ring-shaped trap discussed above is the case closest to the theoretical discussions of the superfluid fraction [12], and the simplest to present. However, our method also applies to a quasi-2D or 3D gas, provided the optical fields are such that the atoms always remain in the lowest energy band. As an illustration we consider a scenario in which two hyperfine levels [15], labelled $\uparrow$ and $\downarrow$, are coupled by the L-G beams propagating along $z$. In the $\left(\psi_{\uparrow}, \psi_{\downarrow}\right)$ basis, we parameterise the lowest energy dressed eigenstate as $\left(e^{i \chi} \sin (\theta / 2), \cos (\theta / 2)\right)$, where $\theta(\boldsymbol{r})$ and $\chi(\boldsymbol{r})$ depend on the local optical field. For beams with angular momentum difference $\Delta \ell$, we take $\chi(\boldsymbol{r})=\Delta \ell \phi$, where $\phi$ is the azimuthal angle around the $z$ axis. Assuming optical fields such that $\theta(\boldsymbol{r})=$ $\alpha r \ll 1$, where $r$ is the radial distance in the $x y$ plane, the lowest energy state experiences an effective vector potential that simulates uniform rotation [8]. The total number of flux quanta inside $r$ is $\Delta \ell(\alpha r / 2)^{2}$ and $\omega_{\text {eff }}=(\hbar / 4 M) \Delta \ell \alpha^{2}[17]$. Computing the leading perturbative corrections to the state, as in (10), one finds $\left|\psi_{\uparrow}\right|^{2}-\left|\psi_{\downarrow}\right|^{2} \simeq \Delta p_{0}+\Delta p^{\prime} r\left\langle p_{\phi}(\boldsymbol{r})\right\rangle$, where $\left\langle p_{\phi}(\boldsymbol{r})\right\rangle$ is the local azimuthal momentum density. Thus, the correction to $\left|\psi_{\uparrow}\right|^{2}-\left|\psi_{\downarrow}\right|^{2}$ depends linearly on the local angular momentum density. The spectroscopic measurement of $\left(N_{\uparrow}-N_{\downarrow}\right) / N$, integrated over the sample, therefore provides a measure of the angular momentum per particle $\langle L\rangle / N$. Comparison of $\langle L\rangle$ with $I_{\mathrm{cl}} \omega_{\text {eff }}$ allows the determination of the normal and superfluid fractions.

In summary, we have proposed a method to measure the superfluid fraction of an ultracold atomic gas. It combines the use of optical beams with non-zero angular momentum to simulate rotation, with a spectroscopic readout of angular momentum. Our observation that light-induced vector potentials create a direct connection between the formal definition of superfluid density and the spin composition of a gas is very general, and we expect it to be applicable to other experimental scenarios.

We thank Jean Dalibard and Mike Gunn for helpful comments. This work was supported by EPSRC Grant Nos. EP/F032773/1 (NRC) and EP/G026823/1 (ZH).

[1] Bose-Einstein Condensation, edited by A. Griffin, D. W. Snoke, and S. Stringari (Cambridge University Press, Cambridge, 1996).

[2] A. J. Leggett, Rev. Mod. Phys. 71, S318 (1999).

[3] C. N. Yang, Rev. Mod. Phys. 34, 694 (1962).

[4] M. H. Anderson et al., Science 269, 198 (1995).

[5] C. Raman et al., Phys. Rev. Lett. 83, 2502 (1999).

[6] C. Ryu et al., Phys. Rev. Lett. 99, 260401 (2007).

[7] K. W. Madison, F. Chevy, W. Wohlleben, and J. Dalibard, Phys. Rev. Lett. 84, 806 (2000)

[8] I. Bloch, J. Dalibard, and W. Zwerger, Rev. Mod. Phys. 80, 885 (2008).

[9] D. R. Nelson and J. M. Kosterlitz, Phys. Rev. Lett. 39, 1201 (1977); D. J. Bishop and J. D. Reppy, Phys. Rev. Lett. 40, 1727 (1978).

[10] E. L. Andronikashvili, Zh. Eksp. Teor. Fiz. 16, 780 (1946).

[11] T.-L. Ho and Q. Zhou, arXiv:0901.0018 (unpublished).

[12] A. J. Leggett, Phys. Rev. Lett. 25, 1543 (1970); A. J. Leggett, J. Stat. Phys. 93, 927 (1998).

[13] L. D. Landau and E. M. Lifshitz, Statistical Physics Pt 1 (Butterworth Heinemann, Oxford, 1981), Vol. 5.

[14] Y.-J. Lin et al., Phys. Rev. Lett. 102, 130401 (2009).

[15] I. B. Spielman, Phys. Rev. A 79, 063613 (2009).

[16] S. Franke-Arnold, L. Allen, and M. Padgett, Laser \& Photon. Rev. 2, 299 (2008).

[17] For stability of the superfluid flow, we require $\omega_{\text {eff }} R \lesssim$ $\hbar / M \xi$ at the largest radius $R$. 